\title{
NATIONAL PARK SERVICE AREAS \\ COOPERATING WITH \\ U.W. -N.P.S. RESEARCH CENTER \\ Montana and Wyoming
}

\begin{abstract}
GLACIER NATIONAL PARK West Glacier, Montana 59936 Superintendent: Robert Haraden Telephone: (406) 888-5411
\end{abstract}

DEVILS TOWER NATIONAL MONUMENT
Devils Tower, Wyoming 82714
Superintendent: Homer A. Robinson
Telephone: $\quad(307) 467-5370$

FORT LARAMIE NATIONAL HISTORIC SITE

Fort Laramie, Wyoming 82212 Superintendent: Gary K. Howe Telephone: (307) 837-2221

FOSSIL BUTTE NATIONAL MONUMENT P. 0. Box 527

Kemmerer, Wyoming 83101

Superintendent: Roger Martin

Telephone: (307) 877-3450

GRAND TETON NATIONAL PARK P. 0. Drawer 170

Moose, Wyoming 83012

Superintendent: Jack Stark

Telephone: (307) 733-2880

JOHN D. ROCKEFELLER JUNIOR, MEMORIAL PARKWAY

$\%$ Grand Teton National Park

P. 0. Drawer 170

Moose, Wyoming 83012

Telephone: (307) 733-2880
YELLOWSTONE NATIONAL PARK

Yellowstone Park, Wyoming 82190

Acting Superintendent: James

Telephone: 9307) 344-7381

Thompson

BIGHORN CANYON NATIONAL RECREATION AREA

P. 0. Box 458

Fort Smith, Montana 59035

Superintendent: William G. Binnewies

Telephone: (406) 666-2412

CUSTER BATTLEFIELD NATIONAL MONUMENT P. 0. Box 39

Crow Agency, Montana 59022

Superintendent: James Court

Telephone: (406) 638-2622

BIG HOLE NATIONAL BATTLEFIELD

P. 0. Box 237

Wisdom, Montana 59761

Superintendent: Alfred Schulmeyer

Telephone: (406) 689-2530

GRANT-KOHRS RANCH NATIONAL HISTORIC SITE

P. 0. Box 790

Deer Lodge, Montana 59722

Superintendent: Jimmy D. Taylor

Telephone: (406) 846-2070 


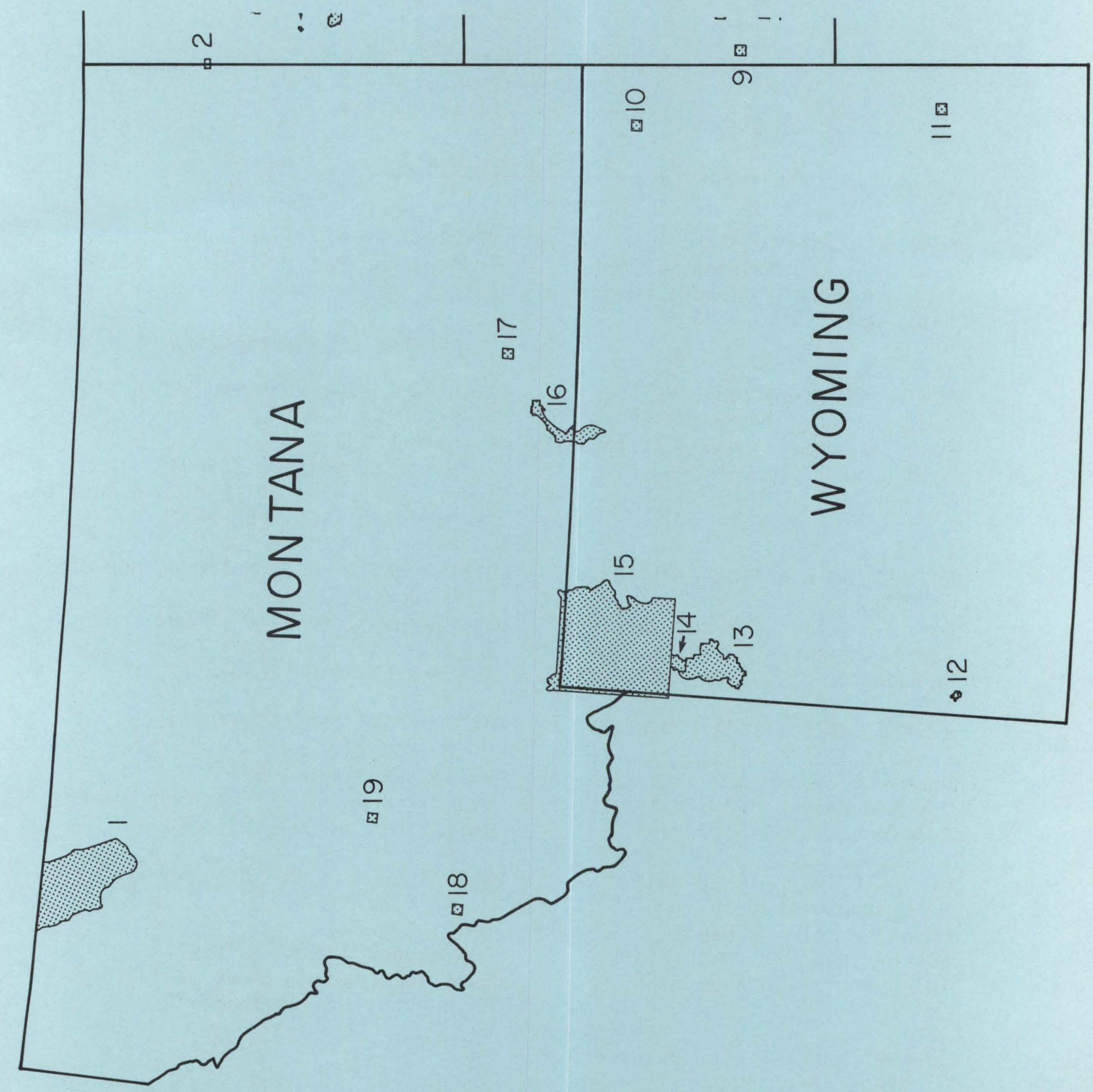




\section{NATIONAL PARK SERVICE AREAS COOPERATING WITH \\ U.W.-N.P.S. RESEARCH CENTER \\ North Dakota and South Dakota}

\section{FORT UNION TRADING POST NATIONAL HISTORIC SITE \\ Buford Route \\ Williston, North Dakota 58801 \\ Area Manager: William E. Wellman \\ Telephone: (701) 572-9083}

THEODORE ROOSEVELT NATIONAL PARK Medora, North Dakota 58645

Superintendent: Harvey D. Wickware Telephone: (701) 623-4466

KNIFE RIVER INDIAN VILLAGES NATIONAL HISTORIC SITE

P. O. Box 175

Stanton, North Dakota 58571

Area Manager: Sandra Hellickson

Telephone: (701) 783-5771

INTERNATIONAL PEACE GARDENS

P. 0. Box 419

\% Theodore Roosevelt National Park

Dunseith, North Dakota 58637

Telephone: (701) 623-4466
BADLANDS NATIONAL MONUMENT

P. 0 . Box 6

Interior, South Dakota 57750

Superintendent: Gilbert Blinn

Telephone: (605) 433-5361

WIND CAVE NATIONAL PARK Hot Springs, South Dakota 57747

Superintendent: Lester $\mathrm{F}$. McClanahan Telephone: (605) 727-2301

MOUNT RUSHMORE NATIONAL MEMORIAL Keystone, South Dakota 57751 Acting Superintendent: Robert Rayer Telephone: (605) 574-2523

JEWEL CAVE NATIONAL MONUMENT Custer, South Dakota 57730

Management Assistant: Dennis L.
Telephone: (605) 673-2288

Di tmanson 


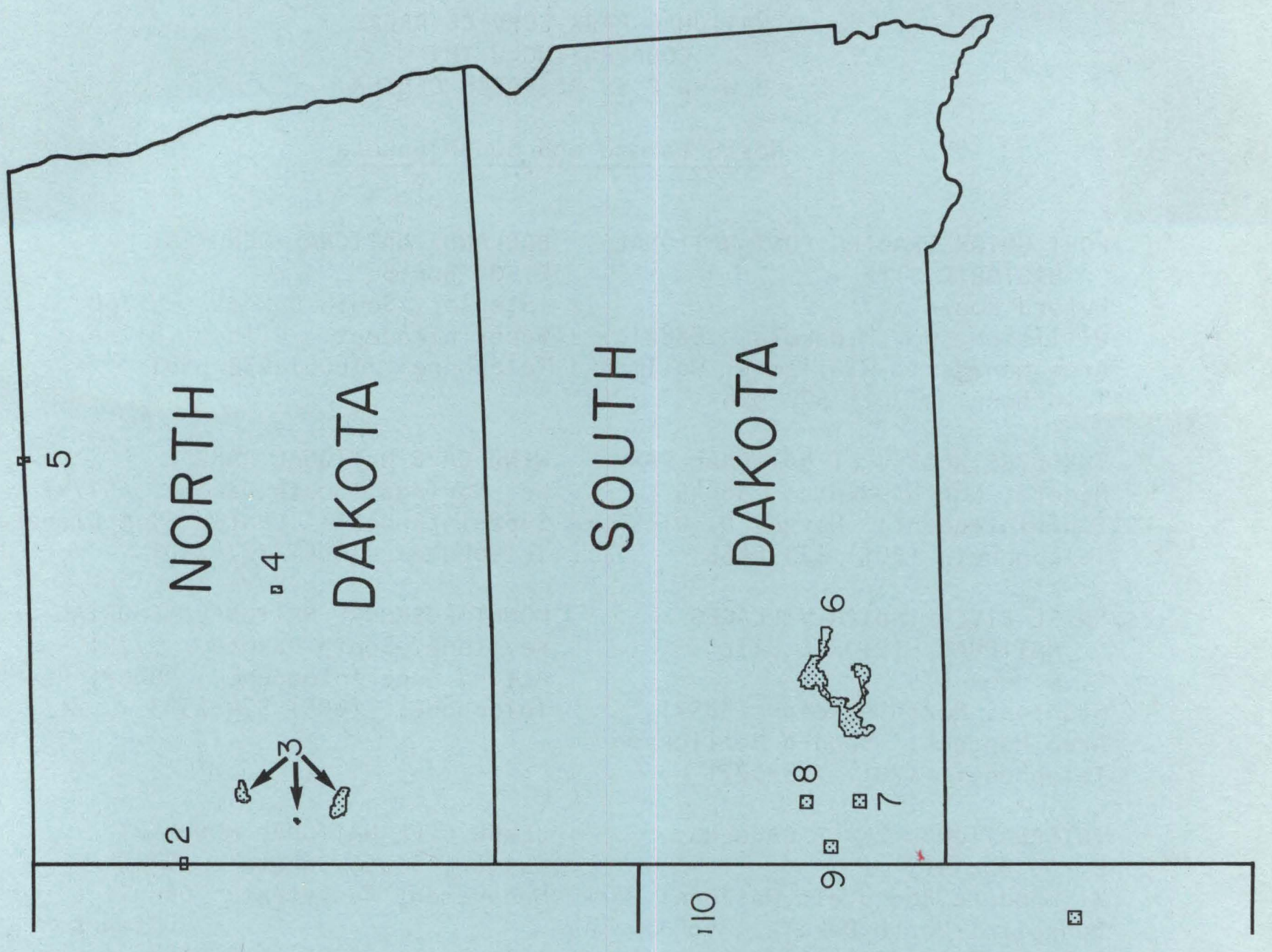

